\title{
Smart Assistant for Accident Prevention and Rescue
}

\author{
Pagadala Yaswanth ${ }^{1}$, Nukala Ram Kumar ${ }^{2}$, Rathor MukeshKumar ${ }^{3}$, Kanwaljeet Singh ${ }^{4}$ \\ Department of Electronics and Communication Engineering \\ Lovely Professional University, Jalandhar, Punjab
}
yaswanthpagadala22v@gmail.com ${ }^{1}$, mail.ramkumarnukala@gmail.com², mukeshrathodm999@gmail.com³, *kamal1997@gmail.com ${ }^{4}$

\begin{abstract}
:
Travelling is playing a vital role in human life, now it has turned to be dangerous due to accidents. In a survey, Govt declared that more than 1.5 lakhs people are expiring in a year via mishap. More-over in the reported death cases two-third victims die due to late arrival of rescue team. In our project Prevention with alcohol sensor \& we are interfacing GSM, GPS, Vibration sensor to know the accident occurrence and place of occurrence and sending message to the rescue team to save the victims as soon as possible. If the route is not visible (due to fog) through Ultrasonic sensor we can drive safely to our destination.
\end{abstract}

Keywords: GPS, GSM, Accelerometer, Arduino, Ultrasonic sensor, Alcohol sensor. 


\section{INTRODUCTION:}

Road Transport system help's people to move everyplace they want to. In the $21^{\text {st }}$ century, Development in transport System strengthen day by day. Transportation is a boon for the human kind but fear is, it is not to be transformed into a bane, because of high speed and neglecting the traffic rules. In this Digital life no of accidents increased to 437,396 resulting in the death of 154,732 people in our country as per the 2019 statistics. Well, if we go through the world statistics, for every 25 seconds 1 person is going to dead. From Statistics it is also proved that people who face accident die manually due to delayed rescue operation. To overcome this problem, we introduced Vibration sensor, GSM and GPS interface to detect the accident and location of the accident occurred place, to rescue the victims as quickly as possible. Alcohol detection sensor interfaced to switch off the vehicle engine when the limit has been crossed. And Ultrasonic sensor used to find the route when our front lights are not working properly.

\section{LITERATURE SURVEY:}

[1] In the Project alcohol sensor (MQ-3) used to detect the alcohol limit to allow access to drive a Vehicle. The Sensor will detect the alcohol content from human breath and information passed to microcontroller. Then Microcontroller decides to give the access or not. If the limit is beyond the fixed value then the vehicle won't start. [2] Addition to that, eye flickering sensor helps allot to locate the flickering of eye is normal or not. While driving if the user eye is not open after few seconds (we can fix time manually) then one beep sound will start. It helps to stay tuned to driving or giving intimation that "I am not at all able to drive the vehicle." We prefer to attach this sensor to our Spectacles. [3] Here when the accident occurs then GSM sends location with the help of GPS and "DANGER" message to the nearest hospitals (we need to keep on updating the number) to rescue the victim. LM393 and MEMS helps to detect the accident occured. The Vibrations sent from the sensor to microcontroller then the actual process starts. And also the device sends the angle of roll over of the vehicle via message. [4] The project was designed using Bluetooth and android application. The accelerometer detects the axial position of the vehicle. If the position is greater than or less than the critical value then the microcontroller activates the Bluetooth which will note the heartbeat values using IR sensor and the another Bluetooth module will activate the mobile application which will send the GPS location to the nearest hospital and to the registered mobile number. [5] The project was designed using GSM and GPS modules using arm microcontroller. The project keep on checking the values of the axis using accelerometer. If the system is in normal condition it keeps on working. If any changes happened the microcontroller asks the values of the axis and wait for 5 seconds for reset button to be pressed. If it is not stopped then microcontroller will take's the latitude and longitude values and send an aware message to the registered mobile number present in program with latitude and longitude values.[6] Another way of doing this project is by using Machine Learning. The combination of IOT devices and Machine Learning techniques are used to detect the accident. Using Machine Learning techniques the vehicles 
are being monitored every minute. It is a costly method to do. The accident that was detected is informed to the ambulances and the information about traffic congestion is also given to the ambulance drivers. [7] A media known as GSM helps to pass the information to the required one. We can track and navigate the vehicles via GPS. The Rescue team will get the GPS location through GSM. [8] Ultra-Sonic is Associate in Nursing instrument used for measurement the gap to Associate in Nursing object by the employment of inaudible sound waves. The device consists of 2 vital components-Triggers and Echo. Trigger is sort of a transmitter, it sends a wave from the device. Echo could be a receiver. The wave transmitted from the trigger gets mirrored back when touching the article and echo receives that wave, so conniving the gap. it's wont to live the gap between 2 cars to create good distance from the article. [9] Occurrence of the accident detects by the sensor Piezoelectric and intimates to MCU. The Physical quantities pressure and acceleration measured by Piezoelectric sensor. The Principle to find both Physical quantities are similar but slight difference in applying force on the sensing element. [10] In Past days, the accident information can be transmitted but the Occurred place of the accident can't be identified by the rescue team. The airbag system was introduced in 1968 through difficult terrain, pulling a heavy load at slow speed off a slope, crawling out of soft dirt. The system till now deals with only two sensors where the sensor is used to detect the angle (MEMS) and to find any change in the vehicle(Vibration sensor). The main control unit we use Arduino to detect and alert when an accident occurs either in a Message system or a display system. The vibration sensor plays a vital role here then the sensor receives the vibrations of the vehicle, which turns into functions as an accident Occurence device. [11] Arduino gets information from all the sensors and delivers the message to the receiver mobile. GSM helps to make the connection between tower and the Rescue team mobile. The GPS gets the coordinates in latitude and longitudes and the received data is passed to Arduino first and the information is transmitted to the contact given in program via the GSM module. [12]A piezoelectric sensor detects the first occurrence of the accident and hints at the MCU. The physical quantities commonly measured are Acceleration and Pressure by the Piezoelectric sensor.

\section{PROPOSED WORK:}

A period in past, the accident can't be prevented or the help the reach the location in time and it is very hard to find the location of the accident. To avoid causalities, Airbags are used in all vehicle airbags for safety and safety driving. The airbag system was introduced in 1968 through difficult terrain, pulling a heavy load at slow speed off a slope, crawling out of soft dirt. Most of the systems proposed to inform the accident Happened Somewhere. But all these methods are related to after accidents. But we are working to solve these issues. Firstly, we are looking to avoid the accidents. The most reason of accident is the driver is driving the vehicle passing the alcohol drunk threshold. So, we are using the MQ3 sensor which is alcohol gas detection senor which senses the alcohol quantity when the driver breaths air on it before driving the vehicle. If the threshold is under control the engine starts. Otherwise, the engine stops, and he cannot drive the vehicle. And the Second reason of the accidents is distance maintenance between vehicles. To avoid this, we are using ultra sonic sensor to maintain the distance between the vehicles. If the distance cross the limit and alert will be sent to the driver and device waits for 10 secs and if the distance is still not maintained 
then the engine stops immediately, and break will on automatically. These two methods avoid the most cases of accidents but the ensure the safety of the travelers we are using accelerometer sensor to detect the accident immediately and which the help of Arduino, GPS and GSM we can send the location and alert message to the nearby hospitals and police station and also to the particular mobile numbers stored in the SIM of GSM. Hence, help can reach the causalities in time at any location.

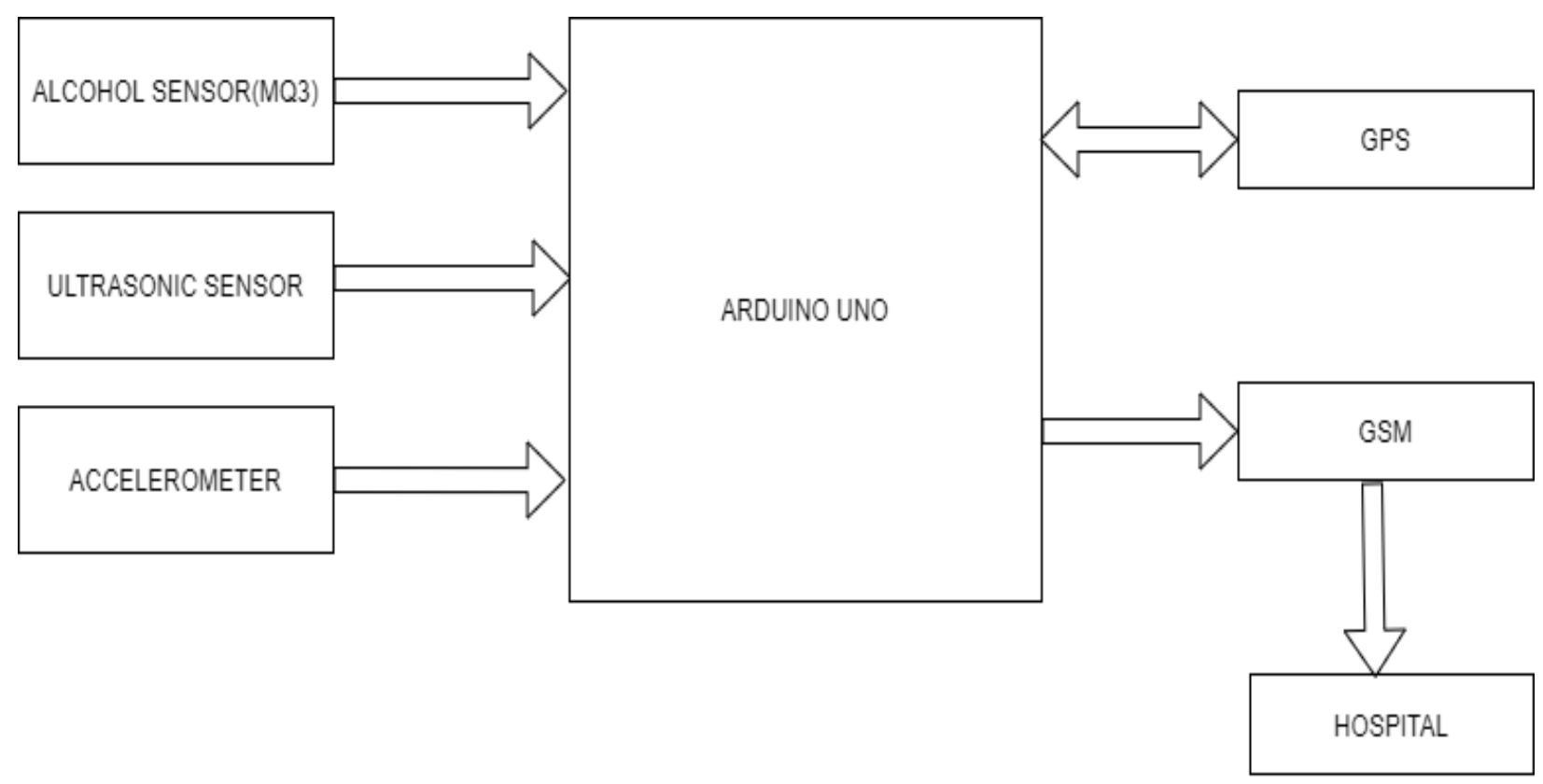

Fig 3.1. Proposed Work Block Diagram

\section{COMPONENTS DESCRIPTION:}

\subsection{ARDUINO UNO}

Arduino UNO board is based on ATmega328P Microcontroller. We can connect the microcontroller board to the computer using USB connection. It is easy to code the microcontroller and burn the program into the microcontroller. A software named Arduino IDE is used to write the code and burn the code o microcontroller using USB connection. We can easily program and edit our program in IDE software. It has flash memory of $32 \mathrm{~KB}$, SRAM of $2 \mathrm{~KB}$ and EPROM of $1 \mathrm{~KB}$.

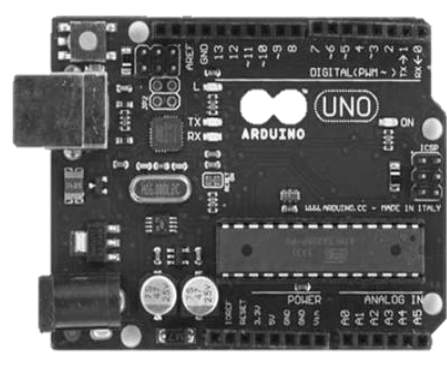

Fig 4.1 Arduino UNO 


\subsection{ACCELEROMETER (ADXL335)}

Accelerometers are wide utilized in cost-sensitive and for tilt sensing applications like mobile devices, play systems, sports. A lower-powered MEMS measuring device is meant on the ADXL335 module, that includes a full sensing vary of \pm three $\mathrm{g}$. It measures the static acceleration thanks to gravity in tilt-sensing applications, together with that dynamic acceleration ensuing from motion, vibration or shock. However $5 \mathrm{~V}$ microcontrollers like Arduino interfacing with associate degree onboard $3 \mathrm{~V}$ regulator makes it is an ideal alternative for the system. It associates degree
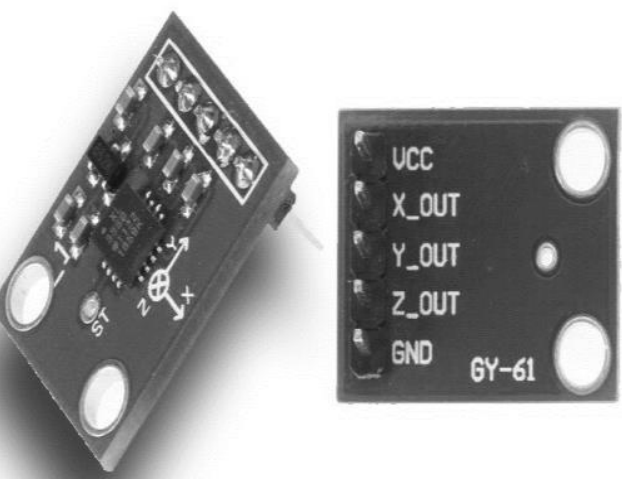
integrated temperature detector. It is a 5 pin module with VCC,

Fig 4.2 Accelerometer GND, X-out, Y-out, Z-out.

\subsection{GSM MODULE (SIM900A)}

The SIM900A may be pronto as a GSM/GPRS module, used in several mobile phones. The module must be used for developing Internet of things (IOT) and embedded applications also. SIM900A might be a dual-band GSM/GPRS engine that works on frequencies. SIM900A options GPRS multi-slot category 10/class eight and supports the GPRS writing schemes. It supports Mike, audio and speaker input. It options a time period clock. It supports both UART and communication through commands. It supports one SIM card slot.

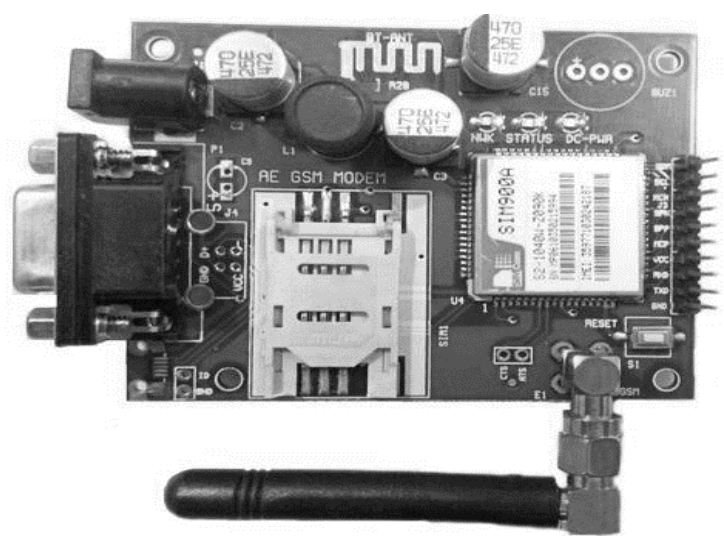

Fig 4.3 GSM Module 


\subsection{GPS MODULE (NEO-6M)}

Global Positioning System allows anyone to track the location of any GPS device from anywhere on the earth. It is a satellite-based system that utilizes ground stations and satellites to find its location on Earth. For accuracy purpose GPS receivers need to receive data at least from 4 satellites. GPS receiver module gives output in standard NMEA string format, which provides output serially through Tx pin with default baud rate 9600. GPS module is a 4-pin module, which provides information about latitude, longitude and time values from GPS. It mostly operated at a voltage of $3.3 \mathrm{~V}$ or $5 \mathrm{~V}$. The module comes with a patch antenna having -161 decibel $(\mathrm{dB})$

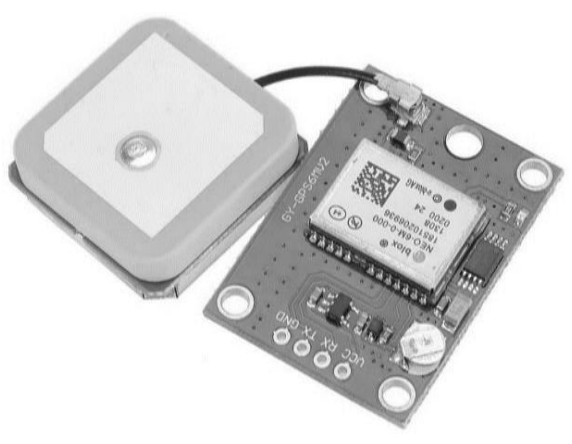

Fig 4.4 GPS Module sensitivity.

\subsection{ULTRASONIC SENSOR (HCSR04)}

The Ultrasonic Sensor is used to detect the object in front of it and can be used to measure the distance between. It uses ultrasonic sound waves for functioning. It emits the sound waves and notes time of emission and if an object is present in front of it then the object reflects the sound waves which then received by the sensor and notes the time of receiving. Hence using time and frequency we can measure the distance between the object and source. The Sensor have 4 pins as echo, trig, Vcc and Gnd. It is operated at $5 \mathrm{~V}$. Distance can be calculated using the formula below:

$$
\mathrm{D}=1 / 2 * \mathrm{~T} * \mathrm{C}
$$

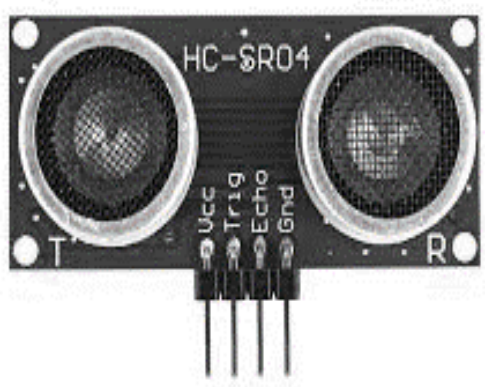

Fig 4.5 Ultrasonic Sensor

\subsection{ALCOHOL DETECTOR MQ3}

This Alcohol Gas detector MQ-3 may be a low-priced semiconductor detector that detects alcohol gases. The fabric is created from $\mathrm{SnO} 2$, that has lower physical phenomenon in traditional air. Once it feels the presence of alcohol in air the physical phenomenon will increase. it's an honest resistance to disturbances thanks to smoke, vapor and fuel. it's a awfully sensible sensitivity and fast latency. It needs straightforward drive circuit, that consists of 1 resistance. This detector works with the facility provide of zero - three V ADC. It is fast responsive and highly sensitive. It requires simple drive circuit.

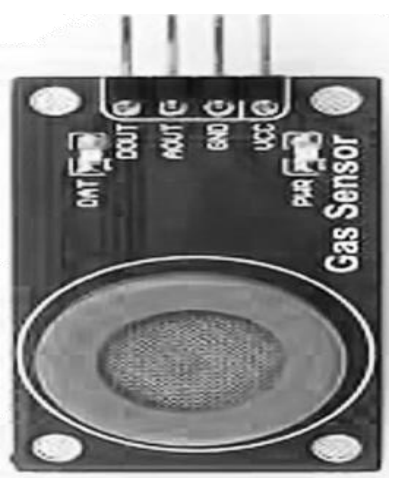

Fig 4.6 Alcohol Sensor 


\section{Methodology}

The complete device can help to decrease the chances of accidents and, we can see the vast decline of accidents and deaths due to accidents when it reaches the normal usage in society. To avoid different cause of accidents we are implementing to 3 step measure.

\section{Step1:}

Major accidents are due to drunk and drive. To avoid this, we are using the breath analyzer with MQ3 sensor where the driver has to breath into the analyzer, If the alcohol content is above 400ppm then the vehicle engine stops and cannot drive the vehicle. This will reduce the major accidents.

\section{Step2:}

The second major reason for occurrence of accidents is that distance maintenance between the vehicles. In traffic places or not non-traffic places accidents occur due to not maintenance of distance. So, to maintain the distance we are using ultra sonic sensor to measure the distance between the vehicles if the distance less than $\mathbf{3}$ meters and alert will be sent to driver. If this is implemented in the society the major accidents in populated/developed cities will be reduced.

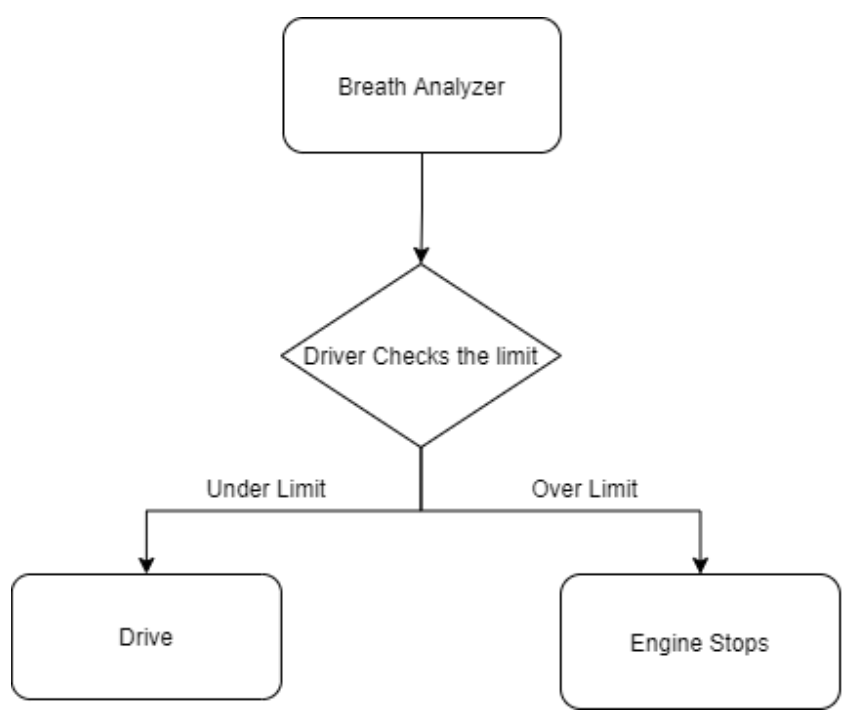

Fig 5.1 Flow chart of step1

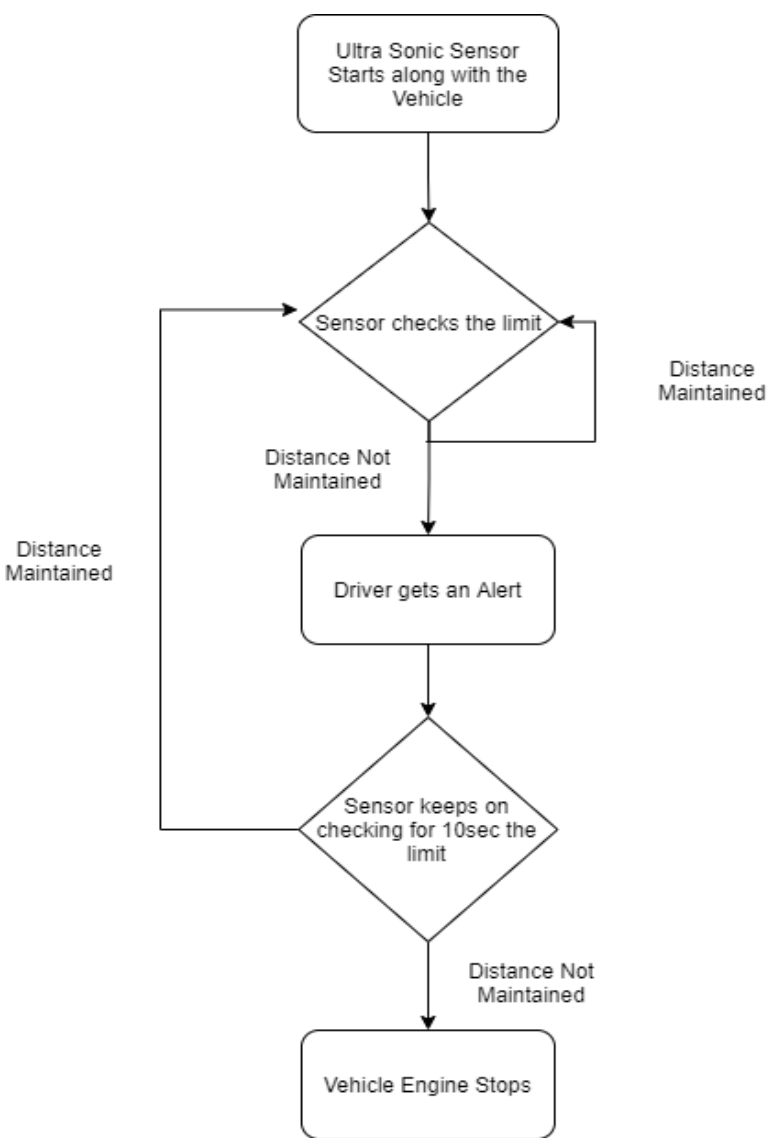

Fig 5.2 Flow Chart of step2 


\section{Step3:}

The above steps will reduce the major cases of the accidents but If an accident occurs even with these measures, we are using this method that help would reach the place immediately. We are using Arduino, Accelerometer sensor, GPS, and GSM to detect the accident using XYZ co-ordinates to determine the gravity acting on the vehicle. When accident happens the severity is sensed and send to Arduino with help of GPS, Arduino gets the accident occurred location with the help of GSM the "help" message sends to the numbers stored in the SIM in GSM.

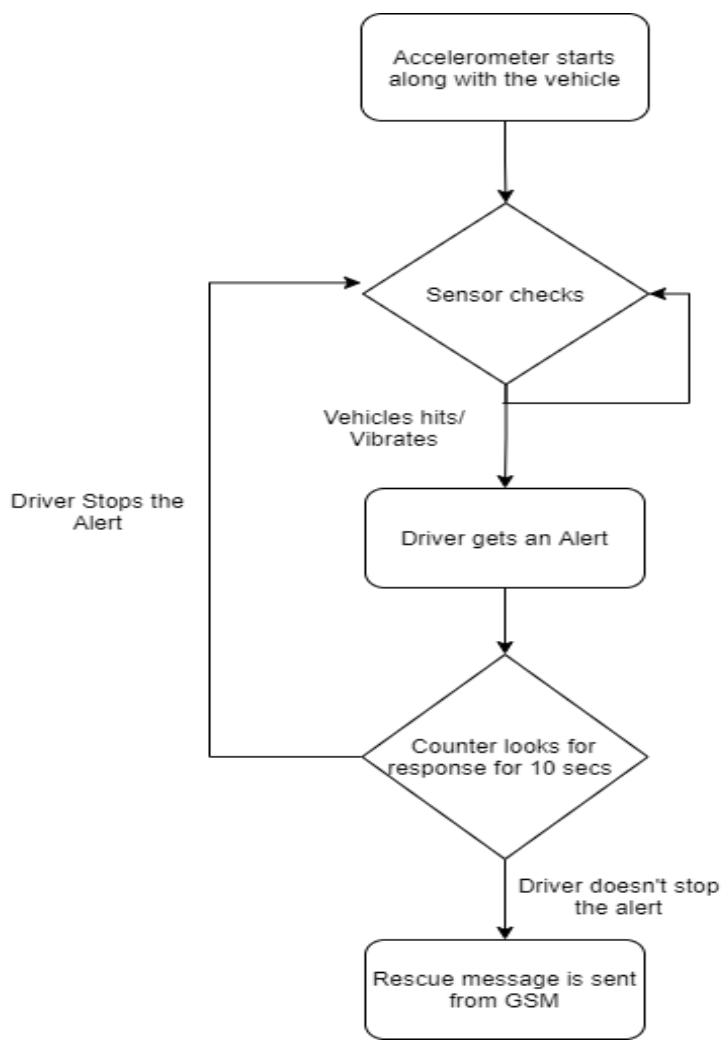

Normal

Fig 5.3 Flow chart of Step3

\section{CIRCUIT}

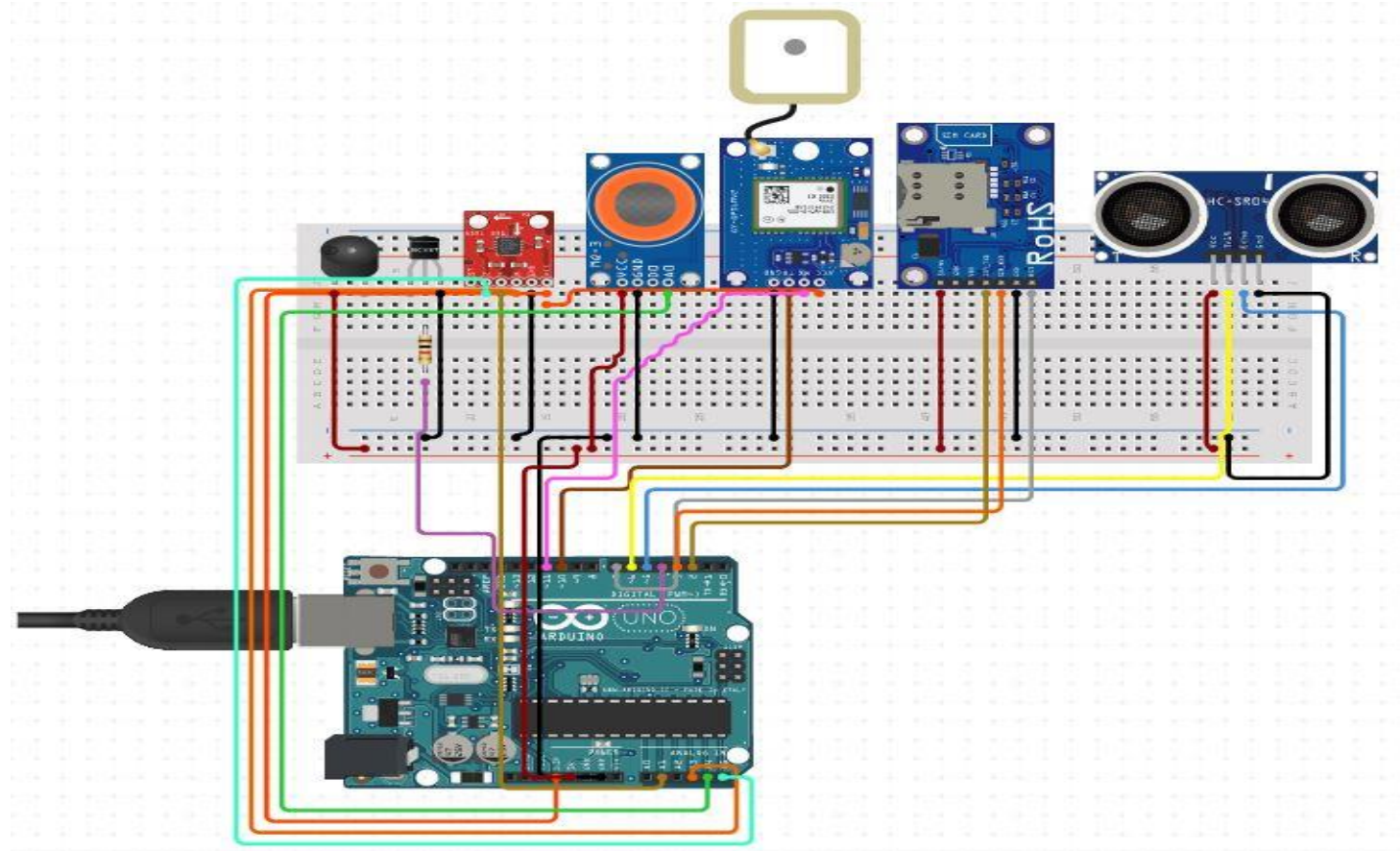

Fig 6.1 Circuit Diagram 


\section{RESULT AND DISCUSSION:}

The main idea of our projects is to prevent the accidents and rescue those hit with an accident. To fulfil our ideas, we across the solution of "Smart Assistant for Accident Prevention and Rescue" with the help of a smart assistant we can avoid the accidents due to drunk and drive and also we can avoid the accidents in heavy traffic areas. If we can implement these two technologies and rules in the society, we can prevent major amount of accidents. Major deaths of people due to accident is that the help couldn't reach in time. To help the rescue team reach early and save lives with help of our smart assistant we can sense the accident and send alert message which consists of accident severity and accident place to the nearest hospitals and family members of the victim.

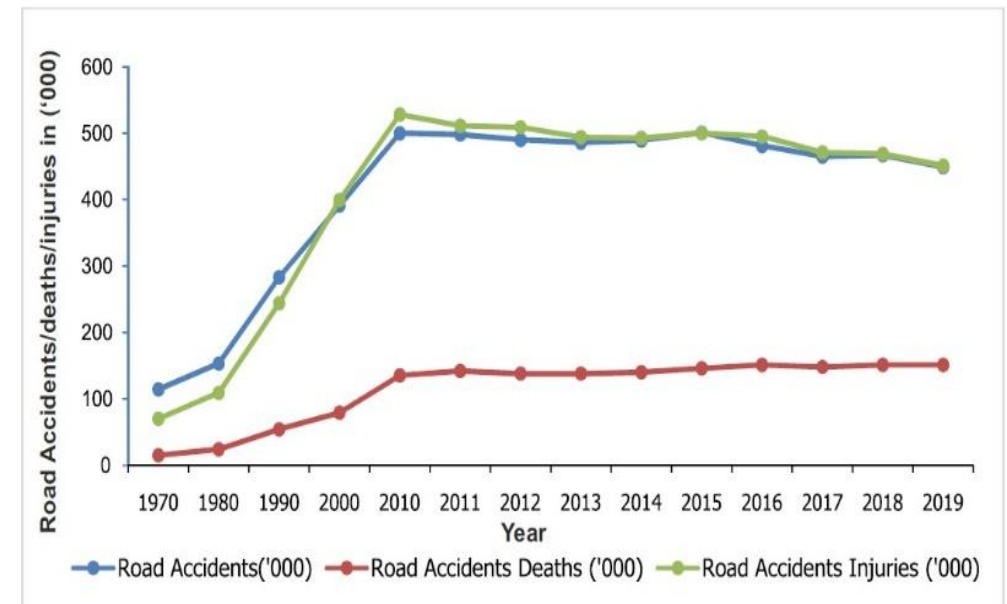

Fig 7.1 Accident Analysis Graph

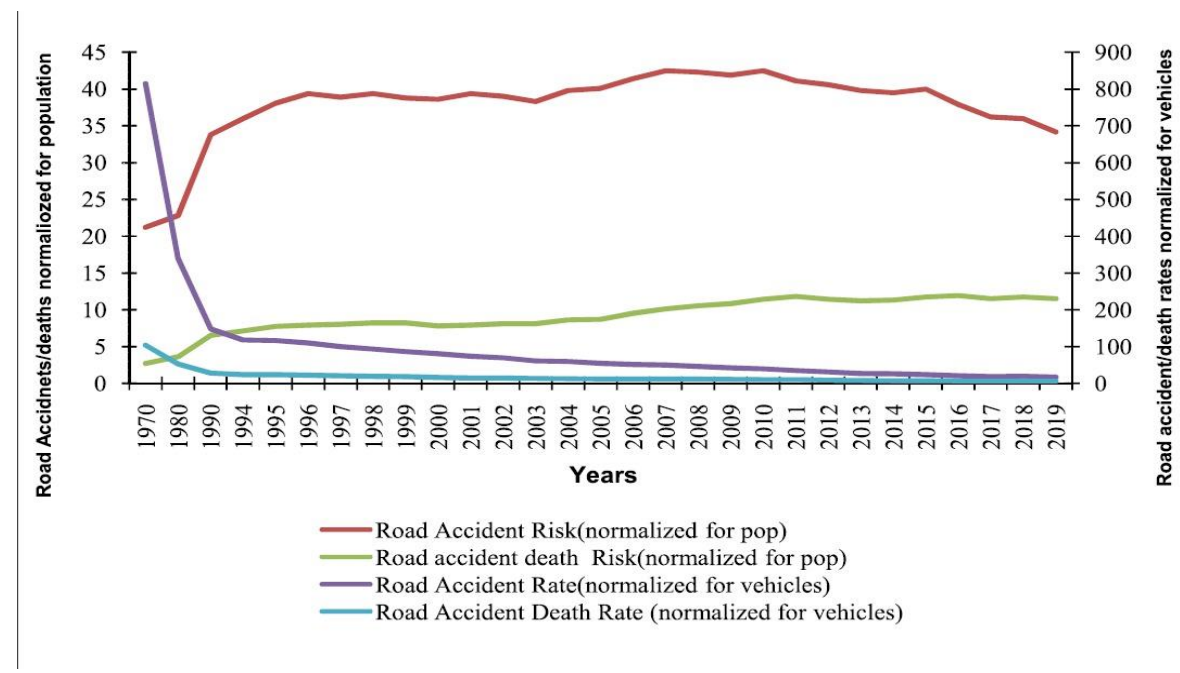

Fig 7.2 Normalized Accident Analysis Graph

Hence our idea of "Smart Assistant for Accident Prevention and Rescue" makes a great impact in preventing accidents and rescuing the victims. 


\section{CONCLUSION:}

As per the report, it can be noted that a variety of tasks have been performed in this field so far. Many performed the tasks of recognizing the crash, finding the accident site, sending the driver a warning letter, etc. The 'Vehicle Accident Avoidance, Monitoring and Tracking System' in this project is designed using GSM and GPS. When an accident happens, the coordinates of the location of accident are received using GPS, and they are sent to registered mobile numbers through a GSM network. This paper not only include the work to detect an accident, but also to avoid it. On one side, the introduction of the vehicle system would lead to higher vehicle prices, but on the other side, it would also increase the chances of being safe on the road and avoiding any mishap. It is noticed that the proposed method is extremely profitable in charge of determining the accident location to provide the immediate rescue to the injured person.

\section{FUTURE SCOPE:}

Day by day, vehicle mishaps are increasing. It is therefore necessary to find a way to reduce these disasters. It can be observed from this paper that a device like this can save many lives. As of now, the device uses a location dependent on GPS and the GSM module uses a warning code. In the future, this framework can be extended by implementing it with Google Maps. Another Improvement could be in the sending module of the message. Aside from only sending the message to the registered numbers, a warning message will also be sent to the ambulance or hospitals available nearby. And we can also improve the accident detection system by predicting the severity of the accident. 


\section{REFERENCE:}

1. Ashokkumar, K., Ch Venkata Deepak, and D. Vamsi Rattaiah Chowdary. "Sign board monitoring and vehicle accident detection system using IoT." IOP Conference Series: Materials Science and Engineering. Vol. 590. No. 1. IOP Publishing, 2019.

2. Choubey, Pankaj Chourasia1 Sakshi, and Riya Verma. "Vehicle Accident Detection, Prevention and Tracking System." (2020).

3. Alshamsi, H., Këpuska, V., \& Alshamsi, H. (2016). Real time vehicle tracking using arduino mega. International Journal of Science and Technology, 5(12), 624.

4. Goud, V. (2012). Vehicle accident automatic detection and remote alarm device. International Journal of Reconfigurable and Embedded Systems, 1(2), 49.

5. Kattukkaran N, George A, Haridas TM. Intelligent accident detection and alert system for emergency medical assistance. In2017 International Conference on Computer Communication and Informatics (ICCCI) 2017 Jan 5 (pp. 1-6). IEEE.

6. Bharath, A. K., Saravanan, M., \& Aravindhan, K. (2019). Literature survey on smart vehicle accident prediction using machinelearning algorithms. International Research Journal of Engineering and Technology, 6(02).

7. Prabha, C., R. Sunitha, and R. Anitha. "Automatic vehicle accident detection and messaging system using GSM and GPS modem." International Journal of Advanced Research in Electrical, Electronics and Instrumentation Engineering 3.7 (2014): 10723-10727.

8. Choubey, P. C. S., \& Verma, R. (2020). Vehicle Accident Detection, Prevention and Tracking System.

9. Amin, Md Syedul, Jubayer Jalil, and Mamun Bin Ibne Reaz. "Accident detection and reporting system using GPS, GPRS and GSM technology." In 2012 International Conference on Informatics, Electronics \& Vision (ICIEV), pp. 640-643. IEEE, 2012.

10. Kattukkaran, N., George, A., \& Haridas, T. M. (2017, January). Intelligent accident detection and alert system for emergency medical assistance. In 2017 International Conference on Computer Communication and Informatics (ICCCI) (pp. 1-6). IEEE.

11. Sharma, S., \& Sebastian, S. (2019). IoT based car accident detection and notification algorithm for general road accidents. International Journal of Electrical \& Computer Engineering (2088-8708), 9(5).

12. Karmokar, P., Bairagi, S., Mondal, A., Nur, F. N., Moon, N. N., Karim, A., \& Yeo, K. C. (2020, August). A Novel IoT based Accident Detection and Rescue System. In 2020 Third International Conference on Smart Systems and Inventive Technology (ICSSIT) (pp. 322-327). IEEE. 